\title{
The experiences of colorectal cancer patients in postoperative recovery: integrative review
}

\author{
Khwanhathai Changyai \\ Royal Thai Air Force Nursing College, Bangkok, Thailand, and \\ Puangpaka Kongvattananon and Chomchuen Somprasert \\ Faculty of Nursing, Thammasat University, Bangkok, Thailand
}

\section{Experiences of colorectal cancer patients}

Received 24 May 2019 Revised 16 August 2019 23 August 2019 Accepted 27 August 2019

\begin{abstract}
Purpose - The purpose of this paper is to synthesize the experiences and needs of colorectal cancer patients during postoperative recovery.

Design/methodology/approach - This study is an integrative review, conducted by CINAHL, PubMed, ScienceDirect and ThaiJO from 2009 to 2019. Ten research articles published have been selected by PRISMA process.

Findings - Results show the experiences of colon cancer patients during postoperative recovery consisted of four themes, namely suffering, decreased activity, ambivalent feeling and need of caring.

Research limitations/implications - The health care personnel should be aware of these experiences and give holistic care in order to let the patients free from unpleasant experiences and receive the responses which really meet their needs.

Originality/value - Knowledge about the experience and needs of colorectal cancer patients after surgery is of great benefit to healthcare providers who are planning the care, so as to achieve complete holistic care in the future research.
\end{abstract}

Keywords Recovery, Experiences, Integrative review, Colorectal cancer, Postoperative

Paper type Literature review

\section{Introduction}

Colorectal cancer is a common cancer of the gastrointestinal tract and is a major cause of global deaths. Currently, the diagnosis of colorectal cancer is increasing among younger populations in Asia and the West[1, 2]. In the USA, according to data from 1974 to 2013, the number of patients with colorectal cancer increased each year and the incidence rate is now very high among the elderly[3]. It is predicted that by 2030 the incidence of colorectal cancer worldwide will increase by 60 percent.

Surgery is still the main treatment method for colorectal cancer patients. Although surgery is an effective treatment approach, colorectal cancer surgery is an abdominal surgery that has a higher tendency to develop complications compared to other types of surgery. After surgery, the patient can experience changes in both mind and body, including postoperative syndrome, anxiety and depression[4]. In many cases of postoperative colon surgery, medical appliances applied postoperatively can cause additional embarrassment, for example, having a stoma attached, and this can cause additional upset[5].

The main goal of postoperative care is to encourage patients to recover. When the recovery process if good, the rate of complications also decreases and this in turn reduces the re-admission rates to hospitals as well as the relevant costs of admission[6].

(C) Khwanhathai Changyai, Puangpaka Kongvattananon and Chomchuen Somprasert. Published in Journal of Health Research. Published by Emerald Publishing Limited. This article is published under the Creative Commons Attribution (CC BY 4.0) licence. Anyone may reproduce, distribute, translate and create derivative works of this article (for both commercial and non-commercial purposes), subject to full attribution to the original publication and authors. The full terms of this licence may be seen at $\mathrm{http} / / /$ creativecommons.org/licences/by/4.0/legalcode

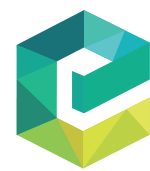

Journal of Health Research Vol. 34 No. 3,2020 pp. $259-269$

Emerald Publishing Limited e-ISSN: 2586-940X p-ISSN: 0857-4421 DOI 10.1108/JHR-05-2019-0103 
JHR

34,3

Moreover, patients can return to performing their daily activities more smoothly. It is essential that care providers understand all aspects of the experiences of patients after colorectal cancer surgery and analyze their needs related to those experiences. In this way, healthcare providers will be able to understand the patient's experience clearly and provide the correct holistic care[7].

This review of existing literature will help to better understand the meaning of the life experiences as well as the needs of colorectal cancer patients after having surgery and during the recovery period. The purpose of this work is to synthesize the qualitative research that describes the experiences of colorectal cancer patients during postoperative recovery in order to obtain a clear conclusion that will be useful for healthcare providers. Such information can be used to make appropriate care plans that meet the needs of specific groups of patients. This could reduce undesirable impacts, promote good recovery and lead to a better quality of life in the long term.

The objective of this integrative review focuses on how patients experience the postoperative period after receiving colorectal cancer surgery, and its purpose is to synthesize colorectal cancer patients' experiences and their needs during the postoperative period.

\section{Methods}

This paper did not involve human subjects. PRISMA's systematic reviews for examining and unifying the literature were utilized. MEDLINE, EBSCO, SCOPUS, CINAHL, PubMed, ScienceDirect and ThaijO were researched from January 1, 2009 to March 1, 2019. The keywords used were related to research articles retrieved from the database suggestions. The journal articles included in this review had to fulfill the following requirements:

(1) published between 2009 and 2019;

(2) primary qualitative research studies;

(3) available in English;

(4) relevant to the experiences of colorectal cancer patients during the postoperative phase; and

(5) limited to the post-discharge period of not less than six months (Figure 1).

\section{Results}

Based on a review of ten studies in Table I, it was indicated that the experiences of colorectal cancer patients in postoperative recovery included four themes: suffering, decreased activity, ambivalent feelings and need of care.

\section{Theme 1: suffering}

Three studies reported that post-surgery, the patient continued to experience changes that occurred from the first stage until leaving the hospital. At first, after recovering from the anesthesia, the patient did not become completely conscious but awareness of his or her surroundings gradually increased. As a result, there was an attempt to pull the abdominal fluid drain out. Some people had negative side effects from the anesthesia, such as feeling tingling and numbness in both legs. Such interference made the patient unable to control his/her balance, leading to walking difficulty. While being cured in the hospital after surgery, the patient, during the early stages, felt weak, exhausted and unable to perform activities on his or her own[9]. Such weakness continued for up to one month, then gradually decreased, and the patient returned to normalcy within six months[16]. On the other hand, the patients that had good pain management did not feel pain because of the wound itself, but undesired feelings were caused by medical procedures such as injections and nasal 


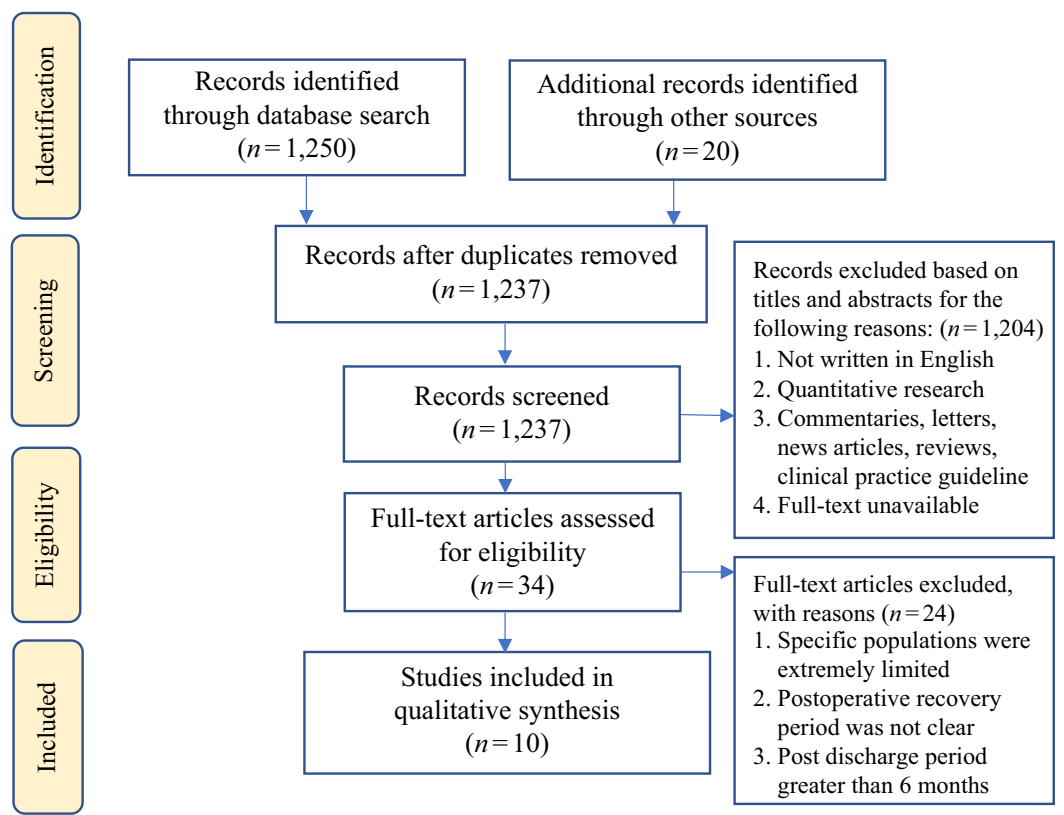

Experiences of
colorectal
cancer patients

261

Figure 1.

PRISMA 2009

flow diagram

adapted to examine

"The Experiences of

Colorectal Cancer

Patients during

Postoperative

Recovery" adapted from[8]

feeding lines[10]. After removing the epidural catheter, patients received pain management by means of morphine intravenous injections with some patients reporting side effects from morphine. After the surgery, it was necessary to refrain from eating food for several days, so patients were likely to lose weight. Some felt uncomfortable and tight in the abdomen even when the drain and drain hose was removed[9]. In addition, it has been found that patients with a drain and drain line felt pain when moving the body so frequently did not want to get out of bed[16]. The illness caused changes in perceptions of taste, causing loss of appetite and a sore throat from intubation and nasal feeding lines. Moreover, the smell of feces or even food was reported disturbing factors that caused poor appetite. Patients that were less able to eat supplements consumed compensatory drinks to allow the body to receive needed energy and nutrients. Some patients felt that their physical conditions were better before surgery[10]. This unusual condition is unfamiliar to patients leading to them wondering when the bad effects would disappear and what would happen next. Consequently, the patients applied various strategies to deal with such symptoms, such as searching for information about symptom self-management, consulting a doctor at the hospital or doing nothing because they thought that the symptoms would disappear with time[11].

\section{Theme 2: decreased activity}

After surgery, one study reported that the obstacle that stopped patients from moving too much was the fear of splitting open their wounds and the uncomfortable feeling caused by the drainage pipe and drain line in the abdomen. Those that underwent abdominoperineal resection surgery developed ulcers in their buttocks, so usually avoided sitting during the first week because of pain. If the drainage was completed, the patient felt more independent and able to move more easily. For one to two days after surgery, patients were not able to take care of their own health because they were weak and[9] exhausted. They relied on others to manage their daily activities. Even when the patients left the hospital, fatigue was 
JHR
34,3

262

Table I.

Summary of studies and their findings of the experiences of colorectal cancer patients during postoperative recovery

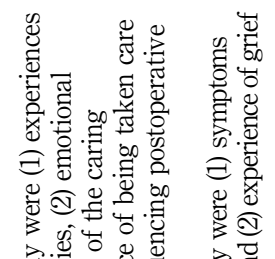

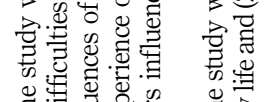

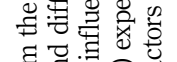

高

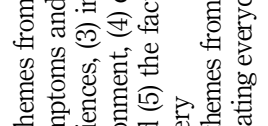

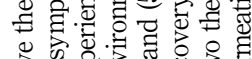

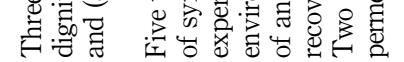

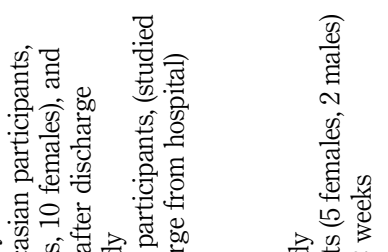

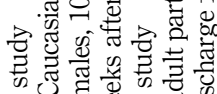

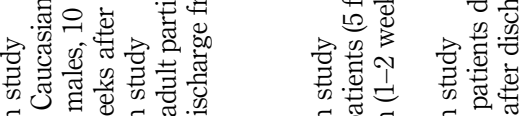

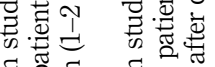

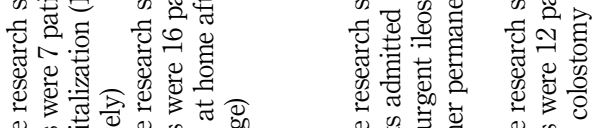

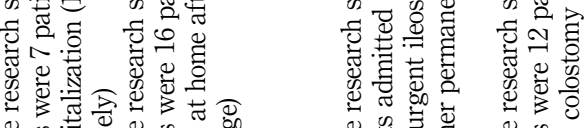

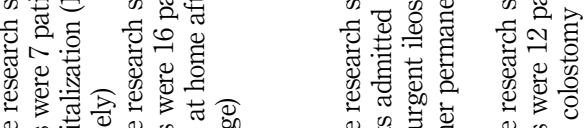

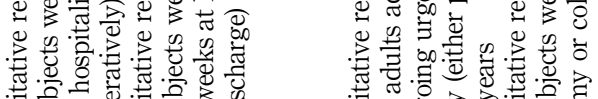

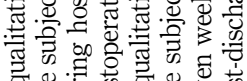

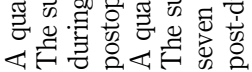

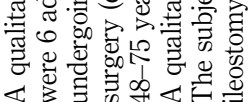

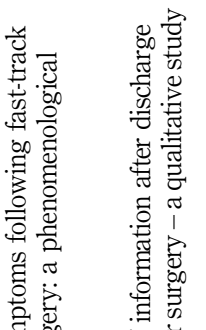

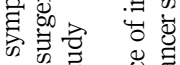

a. के

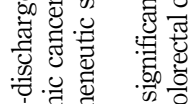

究 융

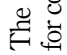

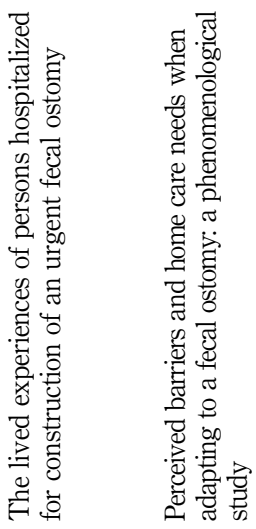

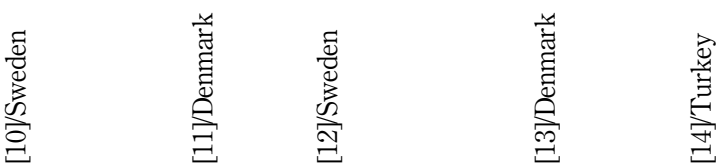

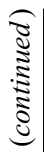

更电

3 害范

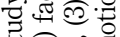

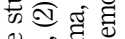

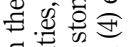

동.

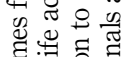

焉要.

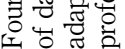

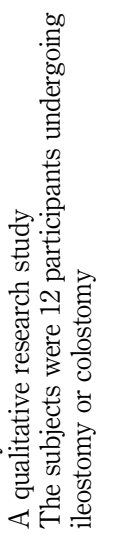

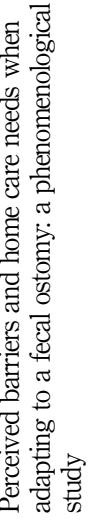




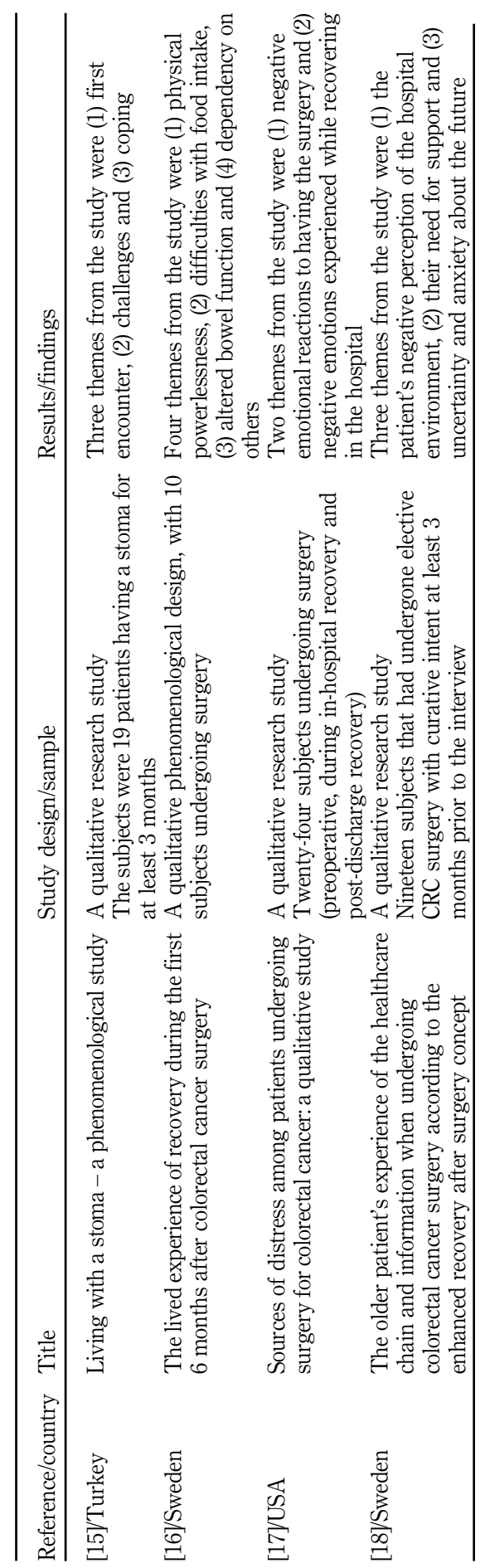

Experiences of colorectal cancer patients

263

Table I. 
JHR

34,3

264

a recurrent symptom, and patients still needed to sleep a lot and did not have much stamina for up to one month after leaving. This left them unable to carry out common tasks like housework or even their daily routines[18]. Three studies reported that when returning home, some patients intended to perform daily activities or exercise on their own. Others were more reluctant and had to be encouraged to do so. However, some patients that had a stoma found it difficult to exercise or perform certain activities[11]. For instance, in some religious activities that required bending or standing for long periods, some patients were reluctant to join such activities for fear that the feces bags would break and possibly embarrass them in public[15].

In addition, five studies about patients that faced stoma problems indicated that they were worried about having sex because they were afraid of pain while performing sexual activities. Their sex drive was then reduced. Furthermore, the stoma could smell bad and perhaps make an uncontrollable noise. Male patients often lost confidence or pride and were concerned that it would cause problems with their respective partners[14].

Patients were aware of existing difficulties and limitations from the early stages of postoperative recovery. They felt disgusted when they saw blood or material emanating from their stoma and were worried about how to manage it $[10,17]$. They were concerned that when the intestine moved, it would hurt. In addition to painful sensations, there could be problems concerning uncontrollable elimination. Feces could leak out[9] because people sometimes could not control their excretion or flatulence or in the case of exercising and exertion, the stool could possibly flow out easily. Moreover, patients would often avoid long journeys, and traveling plans needed to be properly managed. As a result, the mentioned conditions affected normal routines and socializing. Initially, patients had to make significant lifestyle adjustments. Some reported sleeping difficulties due to concerns that the stool would mess up the mattress. Such worries and accidents inevitably affected normal living and spousal relationships[14]. After six months, the digestive system would return to its normal function and the patient would become accustomed to the conditions. They felt that it was a part of their everyday lives[16]. However, some people with a stoma experienced serious problems that impacted on their lives necessitating follow-up appointments with the health team after returning home[14].

\section{Theme 3: ambivalent feelings}

Six studies reported that some patients suffered because they had to undergo urgent surgery without receiving sufficient information about the surgery process or because of inadequate psychological preparation[13, 17]. Although having been informed about the ostomy before the surgery, patients felt shocked when they first saw the stoma in their abdomen. Due to the need to drain the abdomen, the patients did not want to be seen in such a condition[9] and thought it was a burden because they would be weak and dependent on others[13]. During the postoperative period, the patient worried about the biopsy results and the possibility of developing a disease. They normally consulted other patients in order to reduce stress. On the one hand, if they were diagnosed with cancer, the patients were depressed and afraid of losing their normal living routines[11]. Other patients were able to accept this and were ready to monitor the spread and recurrence of the cancer after surgery[10, 17].

Moreover, some felt very depressed when having a stoma and could not accept a permanent ostomy and tended to neglect it. Some of the patients believed that it was a huge burden. It made them feel desperate and some even committed suicide in spite of realizing that the treatment was going to help them live longer. Some patients believed that before having a stoma, their lives were much better[13, 15]. Having a stoma made them lose their confidence and feel embarrassed. When having an artificial anus, the patients normally experienced uncontrollable defecation[14], and especially when sharing a bedroom with the opposite sex, they experienced some embarrassment[9]. However, some patients were able 
to accept this. Some believed that it was something that God gave them and thought that having a stoma was part of the cancer treatment. They were happier with the knowledge that the cancer had been eliminated[14, 15].

In addition, three studies reported that emotional variance caused by either illnesses or medical treatment and unpleasant surroundings created uncomfortable feelings while the patients were staying in the hospital. It was mentioned that while abstaining from food, a patient saw his friends in the same room eating food and then felt hungry. During the night, while the patient was sleeping, he was annoyed by the sounds of the medical devices such as alarms and monitoring systems. Further, when the nurses were at hand to provide their nursing duties, they made a noise, such as walking around and pulling curtains, and this disturbed the patients. A disturbance was also created by fellow patients sharing the same room $[10,18]$. Such an environment caused insomnia and irritation[9] and thus created negative feelings. Many thought that such problems were difficult to deal with when staying in the hospital[18].

\section{Theme 4: need for caring}

After surgery, patients felt exhausted, lacked energy and required more care from nurses and physiotherapy teams in order to facilitate their movements, such as walking or sitting, and needed to be encouraged to move frequently[16]. Patients felt that the hospital was a safe place. They were uncertain of their return home where they would have to take care of themselves or not as well as the health team personnel. Additionally, they did not know how to deal with problems when symptoms occurred. Patients were also impressed by the polite service from the health team. Medical personnel left a favorable impression when responding to the needs of the patients as requested. However, two studies reported that patients were regularly ignored because nursing staff had to carry out other activities besides their nursing work $[10,13]$. At the early stages, patients with a stoma were unable to accept their condition but changed their attitudes with support from doctors and nurses. When returning home, they were often encouraged by family and friends who provided primary assistance and care[14, 15]. With time, patients were able to help themselves[16].

Patients not only needed good care, but also required sufficient information about their health after surgery. After surgery, patients needed immediate confirmation from the doctor. They wanted to know if the tumor was totally removed and wished to be informed about the biopsy result. In cases of cancer results, the patients also wanted to get more information about chemotherapy as well as relevant side effects[18]. The patients worried that some parts of the information were concealed. In order to obtain true information and to ensure the patients' confidence, some patients surfed the internet for additional knowledge[9, 12]. Five studies reported that the information received from staff and the ostomy nurse was insufficient[15], whereas the information received from nurses covered all aspects. Insufficient information included general advice that was not specific to the individual, did not cover certain recommendations, such as the scope of the activities, information about bowel disorders, postoperative wound care, self-care when experiencing abnormal gastrointestinal symptoms[9, 18] and medicine consumption[12], to name a few examples. Yet, problems arose if the advice was not given to the patient at the right time. If they were inattentive when being advised, they were then likely to forget what they were told[14]. One study reported that nursing personnel used difficult terminology, thus hindering the patient's comprehension. Misunderstood information often led to conflicts between patients and healthcare providers; and consequently, made the patients lose trust in the health care team[9]. Therefore, the information needed to be clearly confirmed in written form together with oral communication in order to ensure that the instructions would be followed properly when patients were discharged from the hospital. In addition, it was found that delivering information was a type of one-way communication and there was sometimes no opportunity 
JHR

34,3

266

to ask questions. Discussion with the health team was sometimes only carried out in the morning because the staff had other responsibilities with little time to explain details thoroughly. The elderly, for example, were sometimes not provided with sufficient information because the staff members were in a rush to deliver the information, and not mindful of an elderly person's need for more time to assimilate unfamiliar information. Additionally, the given explanation from the staff members sometimes did not cover the necessities, resulting in anxiety and uncertainty. Instead, they chose to talk to friends that had the same type of surgery and sought knowledge from the internet. Many people wanted to join the health team in planning before discharge so that they were well informed about self-care guidelines. This indicates that caring and information distribution is crucial for patients, and healthcare providers should be aware of this.

\section{Discussion}

The results of the synthesis revealed that the personal experience of the patients during the postoperative colon and rectal cancer resection was primarily related to their physical activity, which shows that during the hospital stay, patients suffered from postoperative symptoms during the early period[10], including pain and fatigue, a common symptom after surgery. While the body experiences fatigue, the patients then suffered from insomnia[19]. Insufficient sleep was common after surgery due to many factors. Side effects from medication and external environments that interfere with sleep also interfered with postoperative recovery[20]. Bowel surgery also contributed to the secretion of hormones that causes decreased appetite; therefore, patients were bored with eating and ate less, resulting in weight loss[21]. These symptoms affected the quality of life during a period of five to seven days after surgery[22]. It was found that during the first week after surgery, the ability to perform most activities was not fully regained so patients have to rely on others[23].

In addition, physical symptoms lead to negative emotions among patients. Most patients after surgery for colorectal cancer were faced with the postoperative psychological experiences of fear and anxiety regarding their illness. These psychological conditions led to a failure to follow instructions and despair in terms of promoting their recovery due to psychological effects.

Having a stoma results in a patient losing confidence with a need to adjust to his/her condition during the postoperative stage. The advice from the health team is therefore important for encouraging people to continue their body movements and to find ways to return to their daily lives[24].

In addition, external factors have an influence on the perception and experience of patients and also have an indirect influence on their recovery, which may be both positive and negative, such as the hospital environment, social support and the healthcare team. Doctors are best qualified to give patients effective strategies for dealing with suffering. Therefore, social support from a doctor together with the healthcare personnel is what the patient desires, and this influences his or her recovery while in the hospital[25]. Furthermore, family support has a positive influence on the patient's behavioral change and postoperative results[26]. It was found that patients who received good social support from family and friends improved in their physical functions and experienced reduced psychological suffering, leading to a better quality of life[27].

Comprehensive and adequate guidance is essential to the process of preparing for the patient's discharge from the hospital. Rehabilitation at home, particularly for those needing ongoing care and follow-up with the healthcare team, benefited from clear guidelines and advice that increased their self-confidence. While in the hospital, the patient needs to know the necessary information, such as advice on pain management, wound care and the limitations of their physical activities. They should ask the health personnel to teach them about self-care in order to improve their health literacy before discharge. In this way, 
patients will be confident that they can take care of themselves effectively during the first one to two weeks after leaving the hospital[26].

The results of this study show that colorectal cancer patients experience a wide range of postoperative recovery effects, both good and negative. Although the needs of colorectal cancer surgery patients may be similar to that of general surgical patients, the data obtained from the synthesis show that there are some distinctive characteristics between the two groups. The experiences and needs of focused patients are different. This is because, in some colorectal cancer cases, the patients might have an ostomy, which changes the body and affects appearance. In some cases, it was found that the recommended actions were insufficient and did not really meet the patients' needs[28]. For this reason, health care professionals should be aware of these special issues and, formulate strategies to better deal with specific patient needs.

In addition, recovery promotion, especially after colorectal cancer surgery, has interested researchers. Currently, the existing guideline for focused patients is the ERAS protocol[29]. However, such a protocol is merely developed with the intention to enhance physical recovery. The promotion of psychological recovery and self-care education procedures are rare. Therefore, it is necessary that the guidelines be especially drawn up in order to manage undesirable experiences and promote patients' education. Furthermore, the guidelines should be included as a part of routine care for specific colorectal cancer surgery patients.

From the results, the colorectal cancer surgery patients would have lots of difficulties in self-adjustment and emotional variance during the early period. If these problems are not managed beforehand, they will continuously impact the patients' quality of living after being discharged[30]. Although a patient appraisal is needed, patient assessment is sometimes conducted from a clinical practitioners' perspective only and does not cover the patient's perspective, so it does not meet the patient's requirements[31]. Therefore, health care personnel should specifically be trained about patient access skills for understanding their experiences and needs, providing counseling and providing necessary information for colorectal cancer surgery patients with ostomy[26]. Moreover, the relatives of patients should be encouraged to participate in experience and needs assessments as they are close to the patient but also able to convey messages to the nurses.

The information received is especially useful for healthcare personnel in terms of patient care. Therefore, it would be interesting for future research to study in-depth strategies for dealing with such experiences and to explore the needs of patients in order to respond to such experiences according to the needs in each area so that a deep understanding of this group of patients can be achieved. In addition, further research should be conducted among the Thai population as most literature reviews are from foreign countries with a different social context. A localized Thai study would result in a needs assessment instrument more suitable for this specific group.

\section{Conclusions}

According to the results of the integrative reviews, it was found that after surgery, the patients with colorectal cancer had to face various experiences during their postoperative recovery, including suffering, decreased activity, ambivalent feelings and the need for caring. This shows that the experiences of persons in this group vary, depending on the individual's perception in different situations. Good recovery is the main goal of caregiving after surgery. Therefore, the health team should pay attention to all factors related to recovery, especially the experiences gained from personal perspectives, which are necessary for creating care plans. Therefore, nurses who are always closest to the patients should develop excellent communication skills but also manage to evaluate each patient's individual experiences in order to develop an improved holistic care system. Ultimately, the goal should be for improved procedures during the recovery period so patients may sooner return to normal and enjoy a better quality of life.

\section{Experiences of colorectal cancer patients}

267 


\section{References}

1. Sheneman DW, Finch JL, Messersmith WA, Leong S, Goodman KA, Davis SL, et al. The impact of young adult colorectal cancer: incidence and trends in Colorado. Colorectal Cancer. 2017; 6(2): 49-56.

2. Chung RY, Tsoi KKF, Kyaw MH, Lui AR, Lai FTT, Sung JJ. A population-based age-period-cohort study of colorectal cancer incidence comparing Asia against the West. J Cancer Epidemiol Prev. 2019; 59: 29-36.

3. Siegel RL, Fedewa SA, Anderson WF, Miller KD, Ma J, Rosenberg PS, et al. Colorectal cancer incidence patterns in the United States, 1974-2013. J Natl Cancer Inst. 2017 Aug; 109(8): 1-10.

4. Yin L, Fan L, Tan R, Yang G, Jiang F, Zhang C, et al. Bowel symptoms and self-care strategies of survivors in the process of restoration after low anterior resection of rectal cancer. BMC Surg. 2018; 18(35): 1-6.

5. Taylor C. Body image concerns after colorectal cancer surgery. Br J Nurs. 2015; 24(S10): S8-14.

6. Bruns ERJ, van Rooijen SJ, Argillander TE, van der Zaag ES, van Grevenstein WMU, van Duijvendijk $\mathrm{P}$, et al. Improving outcomes in oncological colorectal surgery by prehabilitation. Am J Phys Med Rehabil. 2019; 98(3): 231-8.

7. Macmillan CT, Bradshaw E. Holistic assessment of anterior resection syndrome. Gastroenterol Nurs. 2015; 13(3): 18-24.

8. Moher D, Liberati A, Tetzlaff J, Altman DG. Preferred reporting items for systematic reviews and meta-analyses: the PRISMA statement. PLoS Med. 2009; 6(7): 264-270.

9. Worster B, Holmes S. A phenomenological study of the postoperative experiences of patients undergoing surgery for colorectal cancer. Eur J Oncol Nurs. 2009; 13(5): 315-22.

10. Jonsson CA, Stenberg A, Frisman GH. The lived experience of the early postoperative period after colorectal cancer surgery. Eur J Cancer Care (Engl). 2011; 20(2): 248-56.

11. Krogsgaard M, Dreyer P, Egerod I, Jarden M. Post-discharge symptoms following fast-track colonic cancer surgery: a phenomenological hermeneutic study. Springerplus. 2014; 3(276): 1-8.

12. Lithner M, Klefsgard R, Johansson J, Andersson E. The significance of information after discharge for colorectal cancer surgery - a qualitative study. BMC Nurs. 2015; 14(36): 1-8.

13. Herlufsen P, Brodsgaard A. The lived experiences of persons hospitalized for construction of an urgent Fecal Ostomy. J Wound Ostomy Continence Nurs. 2017; 44(6): 557-61.

14. Cengiz B, Bahar Z. Perceived barriers and home care needs when adapting to a Fecal Ostomy: a phenomenological study. J Wound Ostomy Continence Nurs. 2017; 44(1): 63-8.

15. Ceylan H, Vural F. Living with stoma - a phenomenological study. J Nurs Res Pract. 2017; 1(1): 6-12.

16. Jakobsson J, Idvall E, Kumlien C The lived experience of recovery during the first 6 months after colorectal cancer surgery. J Clin Nurs. 2017; 26(23-24): 4498-505.

17. Abelson JS, Chait A, Shen MJ, Charlson M, Dickerman A, Yeo HL. Sources of distress among patients undergoing surgery for colorectal cancer: a qualitative study. J Surg Res. 2018; 226: 140-9.

18. Samuelsson KS, Egenvall M, Klarin I, Lokk J, Gunnarsson U, Iwarzon M. The older patient's experience of the healthcare chain and information when undergoing colorectal cancer surgery according to the enhanced recovery after surgery concept. J Clin Nurs. 2018; 27(7-8): e1580-8.

19. Mota DD, Pimenta CA, Caponero R. Fatigue in colorectal cancer patients: prevalence and associated factors. J Rev Lat Am Enfermagem. 2012; 20(3): 495-503.

20. Su X, Wang DX. Improve postoperative sleep: what can we do? Curr Opin Anaesthesiol. 2018; 31(1): 83-8.

21. Hettiarachchi P, Wickremasinghe AR, Frost GS, Deen KI, Pathirana AA, Murphy KG, et al. Resection of the large bowel suppresses hunger and food intake and modulates gastrointestinal fermentation. Obesity (Silver Spring). 2016; 24(8): 1723-30.

22. Sutti P, Kimpee S, Danaidusadeekul S, Iramaneerat C. Relationship of symptom experiences in symptom cluster's experience with quality of life and symptom management strategies of postoperative colorectal cancer patients. Naresuan Phayao Journal. 2015; 8(2): 107-12. (in Thai). 
23. Lawrence VA, Hazuda HP, Cornell JE, Pederson T, Bradshaw PT, Mulrow CD, et al. Functional independence after major abdominal surgery in the elderly. J Am Coll Surg. 2004; 199(5): 762-72.

24. Russell S. Physical activity and exercise after stoma surgery: overcoming the barriers. Br J Nurs. 2017; 26(5): S20-6.

25. Abelson JS, Chait A, Shen MJ, Charlson M, Dickerman A, Yeo H. Coping strategies among colorectal cancer patients undergoing surgery and the role of the surgeon in mitigating distress: a qualitative study. Surgery. 2019; 165(2): 461-8.

26. Pieper B, Sieggreen M, Freeland B, Kulwicki P, Frattaroli M, Sidor D, et al. Discharge information needs of patients after surgery. J Wound Ostomy Continence Nurs. 2006; 33(3): 281-9.

27. Gonzalez-Saenz de Tejada M, Bilbao A, Bare M, Briones E, Sarasqueta C, Quintana JM, et al. Association between social support, functional status, and change in health-related quality of life and changes in anxiety and depression in colorectal cancer patients. Psychooncology. 2017; 26(9): 1263-9.

28. Lam WW, Law WL, Poon JT, Fong D, Girgis A, Fielding R. A longitudinal study of supportive care needs among Chinese patients awaiting colorectal cancer surgery. Psychooncology. 2016; 25(5): 496-505.

29. Arrick L, Mayson K, Hong T, Warnock G. Enhanced recovery after surgery in colorectal surgery: impact of protocol adherence on patient outcomes. J Clin Anesth. 2019; 55: 7-12.

30. Di Fabio F, Koller M, Nascimbeni R, Talarico C, Salerni B. Long-term outcome after colorectal cancer resection. Patients' self-reported quality of life, sexual dysfunction and surgeons' awareness of patients' needs. Tumori. 2008; 94(1): 30-5.

31. Beaver K, Latif S, Williamson S, Procter D, Sheridan J, Heath J, et al. An exploratory study of the follow-up care needs of patients treated for colorectal cancer. J Clin Nurs. 2010; 19(23-24): 3291-300.

\section{Corresponding author}

Puangpaka Kongvattananon can be contacted at: puangpaka@nurse.tu.ac.th

For instructions on how to order reprints of this article, please visit our website: 